\title{
SALZGEHALTBESTIMMUNGEN \\ DES OBERFLAECHENWASSERS ALS HILFSMITTEL BEI POSITIONSBESTIMMUNGEN AN BORD
}

\author{
voN
}

\section{MARTIN KNUDSEN}

$D^{a}$ der Salzgehalt der Oberfläche des Meeres an verschiedenen Orten ein verschiedener ist, liegt es nahe, die Frage zu erheben, ob Salzgehaltbestimmungen an Bord nicht als Hiifsmittel bei Positionsbestimmungen verwendet werden könnten ${ }^{1}$ ). Die Bedingung für eine solche Verwendung des Salzgehalts des Oberflächenwassers lässt sich leicht aufstellen. Sie liegt darin, dass der Salzgehalt an Orten, die nicht weit voneinander entfernt sind, merkbar variiert, sowie dass die Aenderungen, denen der Salzgehalt an jedem einzelnen Orte im Laufe der Zeit unterliegt, nicht zu gross sind im Verhältnis zu den Salzgehaitverschiedenheiten, die innerhalb des Gebietes vorkommen, das durch die Unsicherheit des gegissten Bestecks angegeben wird. Diese Unsicherheit wird leicht eine grosse, wenn längere Zeit keine astronomische Ortsbestimmung oder Landkennung stattgefunden hat, und somit müsste man die Salzgehaltbestimmung namentlich bei unsichtbarer Witterung zu verwenden suchen.

Eine notwendige Bedingung für die Verwendung des Salzgehalts zur Positionsbestimmung wird natürlich auch sein: eine genaue Kenntnis der Verteilung und Variation des Salzgehalts; bevor man es in dieser Beziehung zu einer vorläufigen Kenntnis gebracht hat, wird es überhaupt nicht möglich sein zu entscheiden, ob Salzgehaltbestimmungen ein so wertvolles Hilfsmittel für die Navigation abgeben können, dass ihre Verwendung einen Vorteil darbietet. Hier ist jedoch in Betracht zu ziehen, dass unsere Hilfsmittel zur Positionsbestimmung an Bord bei unsichtbarer Witterung so gering an Zahl und so unsicher sind, dass jegliche, wenn auch noch so geringe fernere Auskunft von Bedeutung sein muss, da es sich ja darum handelt, den Verlust

1) Prof. Krümmé aus Kiel hat mich darauf aufmerksam gemacht, dass ein derartiger Vorschlag bereits früher gemacht worden ist, und zwar in der Arbeit: „Die Ergebnisse der Untersuchungsfahrten S. M. Knbt. „Drache“. Berlin 1886, Seite 7. 
von Menschenleben und bedeutenden materiellen Werten zu verhindern.

Im folgenden werde iclı die Untersuclıungen erwälınen, dic ich zur Beurteilung der Methode unternommen liabe. Das Ergebnis ist in aller Kürze, dass in vielen Meeresgebieten die Methode kaum praktische Verwendung finden wird, und zwar namentlich nicht in engen Gewässern mit starken und unregelmässig wechselnden Strömungen. In einzelnen Meeresgebieten dagegen reden die Untersuchungen der Methode das Wort, aber an den meisten dieser Orte ist das Beobachtungsmaterial zu geringfügig und unzuverlïssig, um eine sichere Beurteilung zu ermöglichen. Schliesslich macht das untersuchte Meeresgebiet nur einen schr geringen Teil der von den Schiffen befahrenen Meere aus, und solange die Unanwendbarkeit der Methode sich nicht beweisen oder sehr wahrscheinlich machen lässt, meine ich, ist man im Interesse der Menschheit verpflichtet, so umfassende Untersuchungen an\%ustellen, dass man die Methode mit Sicherheit beurteilen kann. Dass Salzgehaltbestimmungen gegenwärtig eine ziemlich umstïndliche Sache sind, was der praktischen Anwendung der Methode Hindernisse bereiten wird, sollte weiteren Untersuchungen keinen Abbruch tun, da man mit fast vollkommener Sicherheit annehmen darf, dass hinlänglich schnelle und bequeme Ausführungen der Bestimmungen schon aufkommen werden, wenn die Methode an und für sich brauchbar ist.

\section{Die Ostsee, die Belte und das siidliche Kattegat}

Diese Gewässer sind von mir in dieser Bezielıung nicht genauer untersucht worden. In der Ostsee scheint das Beobachtungsmaterial mir zur Beurteilung dor Methode zu geringfügig. In den Belten und im siidlichen Kattegat, deren Strömungen so unregelmässig und deren Gewässer so eng sind, und wo man mit so viel Vorteil Tieflotungen benutzen kann, scheint mir die Methode a priori nicht vorteilhaft.

\section{Das Skagerak, die Nordsee und der Kanal}

Die Brauchbarkeit der Methode an den verschiedenen Orten hängt davon $a b$, in wie hohem Grade die Isohalinen $z u$ verschiedenen Zeiten von ihrer mittleren Lage abweichen. Diese Abweichung lässt sich annäherungsweise durch Grössen ausdrücken, die, was die Nordsee und angrenzende Gewässer betrifft, durch früher konstruierte Karten ') über Mittelwert und mittlere Abweichung des Salzgehalts bekannt

1) Conseil permanent international pour l'exploration de la mer. Rapports et Procès-Verbaux des réunions, Vol. VI, pag. XXVI, pl. I \& II. 
sind. Nennen wir die in den Karten dargestellte mittlere Abweichung $\mu$ (in "/o ausgedrückt) und den Abstand (in Seemeilen) zwischen zwei Isohalinen (mit einem Salzgehaltunterschied von $1 \% / 00) \frac{d n}{d \bar{S}}$, so ist dic mittlere Abweichung einer gegebenen Isolıaline (in Seemeilen) von ihrer mittleren Lage gleich dem Produkte $\mu \frac{d n}{d S}$.

Das Resultat der Messungen auf den erwälınten Karten ist:

Nördliches Kattegat und östlicher Teil vom Skagerak $\mu \frac{d n}{d S}=20$ Seemcilen Nordsee 30 Seemeilen SSW von Lindesnaes (rechtweisend) $30 \ldots$ Nordsee 80 Seemeilen W von Stavanger - 35 Horns Riff Leuchtschiff Nordsee 14. Seemeilen $W$ von Helder $\quad-t_{-}$ Kanal 15 Seemeilen NW von Dieppe $\quad$ - 10 -

Aus diesen Zahlen geht hervor, dass man die Methode namentlich an der holländischen Kïste und an der Sïdküste des Kanals versuchen müsste. Um von der Bedeutung der angeführten Zahlen einen Begriff $7 . u$ bekommen, kann man die Verluältnisse in irgend einem der genannten Meeresgebiete genauer untersuchen, z. B. beim Horns Riff Leuchtschiff, von wo seit einer Reihe von Jahıren täglich mit Aräometer ausgeführte Salzgehalthestimmungen vorliegen ${ }^{1}$ ). 20 Seemeilen westlich vom Leuchtschiff ist der mittlere Wert des Salzgehalts $34,00 \% / \omega$, während an den 3620 Observationstagen beim Leuchtschiffe selbst ein so hoher Salzgelialt nur 161 mal vorgekommen ist. Man darf also annchmen, dass ein Schiff, das im Gewässer um Horns Riff einen Sal\%gehalt von $34,00 \%$ beobachtet, sich mit einer Wahrscheinlichkeit von $1 \div \frac{161}{3620}$ oder ca. 0,956 ausserhalb des Leuchitschiffes befindet.

Während $\mu^{\frac{d n}{d S}}$ für Horns Rilf 21 Seemeilen ergah, ist das entsprechende Produkt fïr das Gewässer 14 Seemeilen westlich von Helder nur 6. Hier darf man also weit günstigere Verhältnisse fïr die Anwendbarkeit der Methode zu finden hoffen. Bei llelder (Marsdiep) sind von Januar 1895 bis Januar 1904 zweimal täglich und seitdem dreimal täglich Aräometerbestimmungen ausgeführt worden ${ }^{2}$ ). Bei allen diesen Bestimmungen, deren Anzahl bis Dezember 1906 über 10000 betrug, ist nur 3 mal ein Salzgehalt von über 34 " on beobachtet worden, und dieser Salzgelıalt kommt gewöhnlich in einer Entfernung von ca. 10 Seemeilen von der Küste vor. Ein so hoher Salzgehalt

1) Nautisk-Meteorologisk Aarbog udgivet af det danske meteorologiske Institut.

2) Dr. Hoek's, nach 1903: Dr. RedekE's, Mededeelingen over Visscherij. Helder. seit 1894 . 
wic 34,6\% $1 / 0 \%$ ist in all den 13 Jahren bei Helder kein einziges Mal beobachtet worden, während ein Salzgehalt von $34,6 \%$ in einer Entfernung von 17 Seemeilen von Helder gewöhnlich vorkommt. Hieraus kann man also schliessen, dass ein bei unsichtbarer Witterung von Norden oder Osten kommendes Schiff, welches längs der holländischen Küste fahren will, wenn es einen Salzgehalt von über $34,6 \%$ antrifft, wahrscheinlich ca. 17 Seemeilen vom Lande entfernt ist und gewiss keiner Strandungsgefahr ausgesetzt ist. Es scheint also, dass die Methode hier mit Vorteil angewendet werden kann. Es mag recht wahrscheinlich sein, dass die wenigen bei Helder gemessenen hohen Salzgehalte von Beobachtungsfehlern herrühren, da ja eben die Unrichtigkeit der extremen Werte in einem so grossen Material den lıöchsten Grad der Walırscheinlichkeit hat, wenn Beobachtungs- oder Instrumentfehler überhaupt vorkommen. Ist dies der Fall, so ist die Methode hier noch günstiger, als oben angegeben.

Aehnliche Verhältnisse machen sich wahrscheinlich auch lïngs der übrigen holländischen Küste bis an den Hauptauslauf des Rheins geltend, sowie im Kanal längs der französischen Küste von Cherbourg bis fast nach Calais. Aus den einzelnen Beobachtungen scheint hervorzugehen, dass das salzige Wasser sich an gewissen Orten bisweilen fast ganz bis an die englische Küste des Kanals erstrecken kann, während man an anderen Orten stets eine Süsswasserverbrämung längs der Küste vorfindet. Wenn das salzigste Wasser sich tatsächlich ganz bis an die Küste erstrecken kann, folgt daraus natürlicherweise eine wesentliche Beschränkung der Anwendbarkeit der Methode; dies lässt sich aber nur durch künftige Untersuchungen des Salzgehalts des Küstenwassers im Verhältnis zu dem weiter seewärts vorherrschenden Salzgehalt entscheiden. Auf einer Fahrt vom Breitegrad Helders durch den Kanal wird man aller Wahrscheinlichkeit nach hohe und so ziemlich gleichmässig zunehmende Salzgehalte antreffen. Nimmt der Salzgehalt recht plötzlich ab, bedeutet dies, dass man sich dem Lande nähert, und dann muss man das Lot gebrauchen.

Aus den Werten von $\mu \frac{d n}{d S}$ scheint hervorzugehen, dass kaum die Rede davon sein wird, die Methode im Skagerak und im mittleren Teil der Nordsee anzuwenden. Wie sich die Verhältnisse in der Nähe der Küsten gestalten, lässt sich im allgemeinen aus Mangel an Beobachtungen nicht entscheiden.

\section{Die Gewässer um Island}

Bei Papey, in einer Entfernung von ca. 10 Seemeilen vom Lande, wurden 32 Beobachtungen ausgefühırt, die einen durchschnittlichen Wert von $34,20 \%$ haben. Der höchste dieser Salzgehalte ist 34,72 . 
Weiter vom Lande entfernt und weiter südwärts ist das Wasser salziger, was aus mehreren auf einer Koute zwischen den Färöern und Island ausgefïhrten Reihen von Beobachtungen hervorgeht. Auf 15 Reisen, die zur Beurteilung dieses Verhältnisses benutzt werden können, fand man den Salygehalt von 34,72 durchschnittlich ca. 20 Seemeilen weiter seewärts. In offener See hat man in allen Fällen einen höheren Salzgehalt vorgefunden als bei Papey. Der genannte durchschnittliche Salzgehalt von 34,20 ist nur 1mal in so grosser Entfernung wie 10 Seemeilen von Papey beobaclitet worden. Diese Messungen scheinen also auf die Anwendbarkeit der Methode zu deuten.

Die übrigen bei Island unternommenen Messungen besagen nichts oder sehr wenig rücksichtlich der Brauchbarkeit der Methode, da man bei den der Küste nahe gelegenen Beobachtungen Aufschlüsse über die weiter seewärts herrschenden Salzgehalte vermisst, und umgekehrt.

\section{Das Atlantische Meer}

Längs der Nordküste Schottlands in einer Entfernung von 5-10 Seemeilen vom Lande sind im Laufe von 3 Jahren 67 Salzgehaltbestimmungen ausgeführt worden. Der höchste dieser Salzgehalte ist $35,16 \%$, während der Salzgehalt in grösserer Entfernung vom Lande höher ist. Man kann somit mit grosser Wahrscheinlichkeit den Schluss ziehen, dass ein aus offener See kommendes, sich dem Lande näherndes Schiff bei einem Salzgehalt von über $35,16 \%$ mehr als 10 Seemeilen vom Lande entfernt sein wird. Bei einem Salzgehalt von $34,90 \%$ ist das Schiff wahrscheinlich eben 5-10 Seemeilen vom Lande entfernt. Jedoch scheint das salzige Wasser des Atlantischen Meeres sich bisweilen ganz bis an die Küsten der Hebriden und Shetlandinseln erstrecken zu können, wodurch die Anwendbarkeit der Methode hier wesentlich beschränkt wird. Um dies mit Sicherheit entscheiden zu können, müssten wir indes Salzgehaltbestimmungen von den Küsten besitzen oder, was noch besser wäre, von Orten, die eine solche Entfernung von der Küste haben, dass die Schiffe noch ohne Gefahr dort fahren können.

Was die Westküste von Irland betrifft, kann das salzige atlantische Wasser sich mitunter ganz bis an sie erstrecken, nach Beobachtungen von irischen Leuchtschiffen $z u$ schliessen; hier scheint also die Methode nicht gut anwendbar, falls man nicht statt einer vollen Sicherheit mit einer gewissen Wahrscheinlichkeit vorlieb nehmen will.

Während die Verhältnisse an den französischen Küsten des Atlantischen Meeres in dieser Beziehung unbekannt sind, kann man es für den ganzen östlichen Teil des offenen Atlantischen Meeres als eine Tatsache betrachten, dass $\mu \frac{d n}{d S}$ hohe Werte hat, oder mit anderen 
Worten, dass die Lage der Isohalinen im Laufe der Zeit sehr bedeutend variiert, was der Anwendbarkeit der Methode nicht günstig ist. Ander's verhält es sich dagegen im westlichen 'Teil des Atlantischen Meeı es. Im Laufe von drei Jahren sind von englischen und dänischen Schiffen Proben eingesammelt worden, und diese Proben verteilen sich recht gleichmässig ïber die Monate des Jahres. Auf der Karte Taf. I sind alle innerhalb des Rahmens der Karte fallenden Salzgehalte je durch einen Punkt gekennzeichnet. Ihre Anzahl beträgt ca. 1200. Die roten Punkte, deren Anzahl ca. 650 ist, bezeichnen Salzgehalte von unter 35,00\%, die schwarzen, deren Anzahl ca. 550 ist, Salzgehalte von $35,00 \%$ oder darüber:

Wie man sieht, kommen in einer Zone, die einigermassen der $4000 \mathrm{~m}$-Bodenkurve folgt, rote und schwar\%e Punkte in buntem Gemisch vor. Nördlich der roten Linie kommen nur zwei Salygehalte von über $35,00 \%$ vor. Südlich der schwarzen Linie kommt gar kein Salzgehalt von unter $\mathbf{3 5 , 0 0 \%} \%$ vor. Nimmt man an, dass die beiden hohen Salzgehalte in dem sonst süssen Wasser von Beobachtungsfehlern herrühren, kann man aus der Karte schliessen, dass dic $35,00 \%$ Isohaline sich stets $\%$ wischen der schwar\%en und roten Linie gehalten hat. Einige Salzgehaltbestimmungen (3-4), die nicht mit der Karte übereinstimmten, ergaben sich bei der Durchısicht der Originale als zweifelhaft, indem sie Bemerkungen liatten wie „Korken los“ oder dgl. Diese Angaben sind unberücksichtigt geblieben. Wie man sieht, beträgt der Abstand zwischen der roten und schwarzen Linie westlich vom $42^{\circ}$ Meridian ca. 100 Seemeilen. Ca. $50^{\circ} \mathrm{N}$. Br. und $40^{\circ} \mathrm{W}$. L. entfernen sich die beiden Linien voneinander, inden die schwarze eine östliche, die rote eine nordwestliche Richtung annimmt. Oestlich und nördlich unterliegt also die $35 \%$ Isohaline grossen Schwankungen. Es fragt sich nun, ob man annelımen darf, dass die rote und die schwarze Linie an der richtigen Stelle gezeichnet sind, oder auch nur, dass der Abstand zwischen ihnen einigermassen richtig ist. Hierzu ist zu bemerken: auf Grund der vorliegenden Beobachtungen sind sie wahrscheinlich in zu grosser Entfernung voneinander gezeichmet, denn Schiffe, welche lange Zeit ohne astronomische Beobachtungen haben fahren müssen, können gewiss nicht genau angeben, an welchem Orte die Probe genommen ist. Befindet sich ein Schiff ohne Beobachtungen ein wenig südlich der wirklichen momentanen Lage der $35 \% / 10$ Isohaline, und gibt der Kapitän den Ort 20 Seemeilen zu weit gen Norden an, was sehr leicht geschehen kann, verrïckt sich also die rote Linie 20 Seemeilen gen Norden von ilırem tatsäcchlichen Platz. In ähnlicher Weise kann die schwarze Linie \%u südlich gezeichnet sein, so dass die Entfernung zwischen beiden in Wirklichkeit zu gross gezeichnet ist. Ein solcher systematischer Felıler lïsst sich leider gegenwärtig nicht korrigieren, ohne dass man die Schiffs- 
journale durchsieht und alle Beobachtungen ausschliesst, die nicht in Verbindung mit häufigen astronomischen Beobachtungen ausgeführt sind. Eine solche Arbeit habe ich nicht ausführen können, und es ist wohl auch zweifelhaft, ob das Ergebnis dieser Arbeit der Mühe entsprechen würde, denn bei der langen Aufbewahrung und dem Transport der Proben, bevor sie titriert werden, können sehr gut neue Fehler einlaufen, die sich nicht eliminieren lassen. Das einzige Mittel. um die äussersten Lagen der $35 \%$ Isohaline mit Sicherheit feststellen zu können, bieten meiner Ansicht nach gegenwärtig Titrierungen an Bord, von den Navigateuren selbst ausgeführt, die an diesen Untersuchungen ein Interesse haben. Nur solche Titrierungen dürften dann angewendet werden, die kurz vor oder kurz nach einer sicheren Ortsbestimmung unternommen sind, und wenn eine solche Titrierung eine ungewöhnliche Lage der Isohaline ergibt, müsste die Salzgehaltbestimmung sofort unter der Kontrolle einer frisch geöffneten Normalwasserprobe wiederholt werden. Solche Bestimmungen liessen sich meines Erachtens mit genügender Zuverlässigkeit an Bord der grossen Dampfer ausführen, deren Ausstattung und Mannschaft im allgemeinen vortrefflich sind. - Nach dem jetzt vorhandenen Beobachtungsmaterial muss man annehmen, dass der Abstand zwischen den äussersten Lagen der 35\% Isohaline 60-100 Seemeilen ist, und es liesse sich sehr wohl annehmen, dass ein Navigateur nach mehrtägiger unsichtbarer und stürmischer Witterung für den Aufschluss über den Ort dankbar sein würde, den er durch Salzgehaltbestimmungen erhalten könnte. Dass der Abstand der beiden Linien durch viele Beobachtungen mehrere Jahre hindurch wahrscheinlich vergrössert werden müsste, lässt sich natürlich nicht leugnen. - Wie man sich bei der Benutzung der Tiefenkurven nicht mit einem Lotwurf begnüyt, sondern eine ganze Reihe erfordert, ist es sehr wohl möglich, dass ein entsprechender Vorteil sich durch die Salzgehaltbestimmung erzielen liesse. So ist es möglich, dass man, statt die Lage der hier willkürlich gewählten $35 \%$ Isohaline zu untersuchen, lieber andere Isohalinen benutzen sollte, oder man sollte das Gebiet ausfindig machen, innerhalb dessen Grenzen der Salzgehaltgradient eine gegebene Grösse hat. Kurz, es gibt viele verschiedenartige Untersuchungen, die mit einem wirklich zuverlässigen Material angestellt werden müssten, um die beste Methode zur Anwendung der Salzgehalte zu finden, und bevor eine solche Arbeit ausgeführt ist, scheint es mir sehr gewagt, die Möglichkeit einer vorteilhaften Anwendung von Salzgehaltbestimmungen bei der Navigation leugnen zu wollen. 


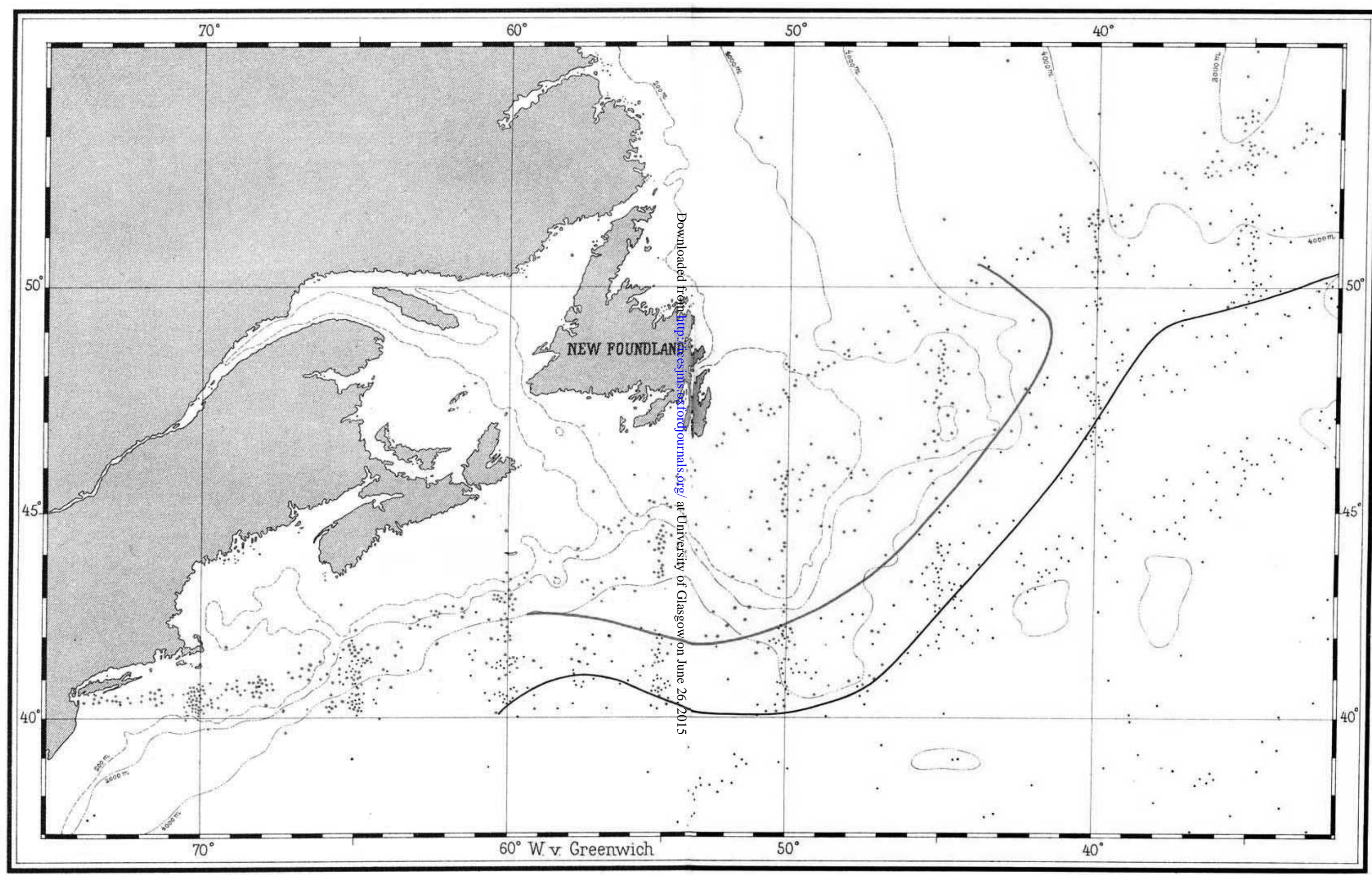

